\title{
IMAGING FOR THE AUSTRALIA TELESCOPE: PRESENT NEEDS AND FUTURE STRATEGIES
}

\author{
RAY P. NORRIS \\ Australia Telescope National Facility*, P.O. Box 76, Epping, NSW 2121, \\ Australia
}

\begin{abstract}
In this paper I outline the strategy we have adopted to produce images from the Australia Telescope. Our main production system is based on AIPS, but we have made several departures from conventional strategy. These departures are amongst several paths that we are exploring in the hope that some will lead to richer astronomical pastures. I will describe the start that we have made on these explorations and our impressions of the scenery along the way.
\end{abstract}

\section{INTRODUCTION}

The Australia Telescope (AT) has several unusual design features, the most obvious of which is that it is located in Australia. The resulting unparalleled access to the Southern skies has considerable implications for the strategy we adopt. First, it means that we put a greater emphasis on Galactic (and hence spectral-line) astronomy than our Northern colleagues. Second, the absence of other powerful synthesis arrays looking at the same part of the sky means that, rather than concentrating on any one observational niche, we need to build a versatile and flexible telescope that will attempt to cover that same range of observational phase space that is so amply covered in the North by powerful telescopes such as the VLA, the VLBA, and MERLIN.

The result of these design goals is a telescope that consists of two parts: a Long Baseline Array, which is a VLBI array organised in collaboration with other Australian institutions, and a Compact Array, located about $600 \mathrm{~km}$ North of Sydney. I will confine this paper to discussion of the Compact Array.

The Compact Array consists of an East-West line of six 22-m antennas spanning $6 \mathrm{~km}$. The antennas may be moved along a rail track, allowing observations to be made in a number of configurations to obtain a large number of $\mathrm{u}-\mathrm{v}$ spacings, and hence a high dynamic range. Each antenna is designed to work up to $115 \mathrm{GHz}$, with a total observable bandwidth up to 1 $\mathrm{GHz}$, and data are transferred over optical fibre to the central building.

Construction of the AT started in 1983, the first image was produced in April 1989, and the AT is now open for proposals from any part of the world.

* The Australia Telescope National Facility is operated in association with the Division of Radiophysics by CSIRO. 
Only five of the six antennas are currently available, and each is presently equipped for only four bands at $20,13,6$, and $3 \mathrm{~cm}$. Nevertheless, the AT is currently used for astronomical observing for nearly half the available time, the remainder being used for system testing and commissioning.

In the rest of this paper I will consider different aspects of our design goals and discuss both the strategies we have adopted and the challenges we have had to meet in so doing.

\section{DESIGN GOALS}

The concept of the AT called, above all, for a versatile instrument. In addition, we have attempted to overcome the inherent slowness of a six-antenna array by allowing simultaneous observing in modes which would require several different observations on other arrays. In practice, this means we have adopted the following design goals:

* wide field mapping capability

* good spectral-line performance

* full polarisation available by default, with high polarisation purity

* wide bandwidth

* simultaneous observing of different frequencies

* simultaneous use of spectral-line and continuum modes.

We have also adopted the strategies of full on-line calibration and the use of AIPS for subsequent data reduction.

\section{CONFIGURATION AND PERFORMANCE}

A typical continuum configuration might use two separate $128-\mathrm{MHz}$ (2 bit) or $256-\mathrm{MHz}$ ( 1 bit) bandwidth bands, with full Stokes parameters available on each. These two bands might be contiguous, to increase the width of the sampling in the $\mathrm{u}-\mathrm{v}$ plane, or they may be well spaced (e.g 6 and $3 \mathrm{~cm}$ ) to measure a spectral index.

A typical spectral-line observer might choose to use the two bands to observe $\mathrm{OH}$ and $\mathrm{HI}$ simultaneously. He can then expect 1024 spectral channels/baseline on each of these with full Stokes parameters, while simultaneously observing a $64-\mathrm{MHz}$ continuum band at both frequencies, with full polarisation over the 32 spectral channels in each of the continuum bands.

Use of wide bandwidths mean that the sensitivity is between one quarter and one half of that of the VLA, although the simultaneous observing modes featured above increase the effective speed. In practice, to obtain a dynamic range of about 1000 , a source will need to be observed with four twelve-hour runs, each in a different configuration.

\section{SPECTRAL-LINE PERFORMANCE}

The spectral-line system features wideband receivers, up to 8000 spectral channels per baseline and simultaneous line and continuum observing. 
The resulting data rate of $200 \mathrm{kbyte} / \mathrm{s}$ (16 Gbyte/day) poses a severe challenge in terms of both the data-transfer rate and also the amount of disk space needed by AIPS users. We have met the data-transfer challenge by adopting a compact and robust data format (RPFITS) and by using Exabyte tapes for data transfer, archive, and storage. In the future we may swap to optical disks if their prices fall sufficiently.

For AIPS processing, we have adopted a goal of allowing each active user about 1 Gbyte of disk space on the Convex $\mathrm{C} 220$, which is therefore equipped with $11 \mathrm{Gbyte}$ of disk. In the near future, as standards stabilise, we hope to use read/write optical disks. Each user will then have one or two 650-Mbyte disks which he will insert in a drive only when he is actively using the system.

\section{BANDWIDTH AND MULTIFREQUENCY SYNTHESIS}

Multifrequency synthesis is the name given to the technique in which a source is observed at several frequencies to yield several tracks in the u-v plane from one baseline. The AT is well suited to multifrequency synthesis by virtue of its inherent spectral-line capability and its wide bandwidth. Multifrequency synthesis will be implemented in three ways on the AT.

First, the wide instantaneous bandwidth of the AT coupled with its spectral-line performance means that each $\mathrm{u}-\mathrm{v}$ track will appear as a broad swath cut through the $\mathrm{u}-\mathrm{v}$ plane. The width of this track (up to $256 \mathrm{MHz}$ ) can be a significant fraction of the observing frequency.

Second, the simultaneous availability of two bands enables two swaths rather than one to be cut by a single baseline in a single observation.

Finally, the wide bandwidth receivers and horns allow rapid switching of the observing frequency. Our experience so far is limited to using a relatively narrow range of wavelengths and assuming a flat spectral index, but we plan to use more sophisticated algorithms in the future.

The large bandwidths used on the AT also raises the problem of interference. This is compounded by the wide bands covered by the feed horns (most of which cover an octave in frequency), and the availability of unprotected spectral-line wavelengths. However, this problem is mitigated by the location of the Australia Telescope in an area less heavily developed than most Northern telescopes.

\section{POLARISATION PERFORMANCE}

Our polarisation strategy aims to make the accurate measurement of all four polarisation parameters (not just linear polarisation) straightforward and routine. One early decision, therefore, was to place feeds on the telescope axis, to avoid the polarisation problems that have plagued off-axis feeds. Thus, all feeds and receivers are mounted in a rotatable turret in the vertex room.

Another decision has been based on the premise that the stability of modern receivers makes the measurement of linear polarisation relatively straightforward, and so the use of circularly polarised feeds is no longer necessary for this purpose. Instead, we have decided to use linearly polarised feeds and tackle the harder question of measuring circular polarisation 
accurately. This use of linearly polarised feeds also allows the use of wide bandwidth polarisers. Rather than relying on the use of astronomical calibrators, we inject a switched noise source into each feed and use a separate correlator to measure the difference in complex gain between the two polarisations. With this technique we hope to be able to calibrate the receiver gains continuously, with primary polarisation calibration being done infrequently from an astronomical source.

\section{WIDE-FIELD MAPPING}

The AT was implemented as an East-West array to facilitate wide-field mapping. A potential problem is that, in addition to the inherent weakness of Selfcal for small numbers of antennas, Selfcal may also be less robust for a linear array than for a two-dimensional array. We are continuing to investigate this effect, but experience so far suggests that, at least at wavelengths of $3 \mathrm{~cm}$ and longer, good a priori calibration plus Selfcal presents no special problems.

We are also investigating the use of mosaicing techniques to map fields even wider than the primary beamwidth. To cope with rapid changes between phase centres, the phase rotators and delays have been designed to allow their settings to be changed in a few milliseconds, and the antennas should be able to move from one beam centre to another in as little as two seconds.

\section{VISUALISATION}

Because the AT is designed as a spectral-line instrument, and because of the large numbers of spectral channels available to the user, we foresee a major challenge in visualisation and interpretation of the data. Current tools such as TVMOVIE are likely to be inadequate for the user with a $(2000 * 2000 *$ 2000)-pixel data cube!

We have therefore embarked on a visualisation project which aims to break through this conceptual barrier separating the spectral-line user from his data. At present we have a pilot project running on a Sun $4 / 370$ with a TAAC imaging accelerator. Techniques being explored include the representation of a data cube as a 3-D image consisting of voxels (3-D pixels) each of which is distinguished by a brightness, a colour, and an opacity. The rotation curve of a galaxy can then be distinguished as a solid object sitting in the middle of a translucent cube, which can then be manipulated, rotated, and sectioned interactively.

\section{CONCLUSION}

I have described here some of the imaging strategies we have chosen to maximise the advantages to be gained from the design goals of the AT. Areas other than imaging are also being explored, such as the use of an expert system for hardware fault monitoring and diagnosis. However, these are early days for the AT, and we are only just starting to explore the ways forward. 
Steven Joersaeter: When do you expect to be able to do $\mathrm{CO}$ and can you say anything on the expected performance?

R. P. Norris: We are currently debating relative priorities on the next receivers to build, but my guess is that it will be a small number of years. I cannot predict at present what their performance will be. 